\title{
Los desafíos de la innovación en Latinoamérica
}

\author{
Alejandro Jiménez ${ }^{1^{*}}$ y Cristian Geldes ${ }^{1}$
}

\section{Introducción}

Actualmente, la innovación es considerada un imperativo estratégico para alcanzar el desarrollo económico y es la mejor opción para enfrentar los desafíos del futuro. Por ello, el estudio y el análisis de sus resultados constituyen una actividad relevante para el mundo académico, para el diseño de políticas públicas y para las revistas que difunden dicha información.

Uno de los referentes más importantes en esta materia es el Índice Global de Innovación (GII)1. Este informe, proporciona valores detallados sobre el desempeño de la innovación en 129 economías del mundo. Sus 80 indicadores aportan una visión amplia de la innovación, lo que incluye al entorno político, la educación, la infraestructura y la sofisticación del mercado entre otros.

Una de las grandes conclusiones del último GII 2019 es que, a pesar de la incertidumbre y la desaceleración de la economía global, el mundo ha experimentado un fuerte aumento de las inversiones en innovación.

En los pasados dos años (2017-2018), el uso de la propiedad intelectual (PI) alcanzó cifras récord. Los gastos globales en I+D se duplicaron entre los años 1996-2016 y crecieron vertiginosamente por sobre el promedio de la economía mundial. En el año 2017, los gastos globales de los gobiernos en I+D en base a su PIB aumentaron en cerca del $5 \%$. Por otra parte, los gastos en I+D de las empresas crecieron en un 6,7\%, el mayor incremento reportado desde el año 2011 (GII, 2019).

Otro dato interesante del informe 2019 es el auge de las economías de ingresos medios. Países como Corea del Sur y China destacan entre los 20 países más innovadores del mundo. Otros países experimentaron un fuerte ascenso como los Emiratos Árabes Unidos $\left(36 .^{\circ}\right)$, Tailandia $\left(43 .^{\circ}\right)$, la India $\left(52 .^{\circ}\right)$, Filipinas $\left(54 .^{\circ}\right)$; que in-gresó dentro de los 55 primeros, y la República Islámica de Irán $\left(61 .^{\circ}\right)$; que se acerca a los 60 primeros (GII 2019).

\section{¿Qué ocurre en Latinoamérica?}

Desafortunadamente, Latinoamérica sigue estancada respecto del desempeño promedio de las economías globales. Solamente Costa
Rica -país de ingresos medios altos- sostiene resultados por encima de sus expectativas económicas. Países de ingresos altos como Chile, Uruguay y Argentina se mantuvieron en concordancia a su nivel de desarrollo. Lo mismo ocurrió con países de ingresos medios altos como Colombia, Brasil y México. Por otro lado, países de ingresos medios bajos como Bolivia, Paraguay y Guatemala, lamentablemente estuvieron por debajo de sus expectativas de crecimiento económico (GII, 2019).

Para JOTMI, la discreta posición de los países latinoamericanos no es una novedad. En el año 2014 publicamos un número especial en el que discutimos con Heitor y col., el rol del cambio tecnológico y las políticas de innovación en Latinoamérica. En aquella oportunidad fuimos muy críticos. La región no ha sido eficiente en promover la innovación en el sector productivo. En términos generales, las políticas de fomento en Latinoamérica no consideran a la empresa como foco del proceso de innovación. Sus instrumentos de apoyo son poco claros, extremadamente burocráticos y descontinuados en el tiempo (Heitor y col., 2014)

También nos planteamos la pregunta ¿puede Latinoamérica avanzar después de una década perdida? Nuestra reflexión nos llevó a argumentar la necesidad de crear redes de valor que potencien el desarrollo inclusivo, las trayectorias de aprendizaje y principalmente la cooperación. Para lo anterior, era necesario realizar inversiones públicas efectivas orientadas a internacionalizar a las universidades y fortalecer la atracción de capital humano avanzado entre otros (Heitor y col., 2014).

Uno de los artículos más citados para en caso chileno, fue el trabajo que publicamos con Horacio Gonzalez en el que criticamos la falta de una política pública destinada a insertar laboralmente a los nuevos investigadores (Gonzalez \& Jiménez, 2014). Actualmente, el principal agente empleador del capital humano avanzado en Chile continúa siendo la universidad $\left(79^{\circ}\right)$. El sector empresarial chileno contrata menos del 5,5\% de los doctorados, mientras que la administración pública menos del 2\% (Encuesta ANIP $^{2}$, 2018). Estos datos no son prometedores y reflejan los pilares más débiles exhibidos por Chile en el último GII 2019: Un insuficiente capital humano e investigación $\left(57^{\circ}\right)$ que produce bajos resultados en tecnología, nuevo conocimiento $\left(61^{\circ}\right)$ y creatividad (66 $)$ (GII, 2019).

\footnotetext{
${ }^{1}$ Global Innovation Index 2019 (GII).

${ }^{2}$ Inserción laboral de investigadores, Informe de resultados: 2da Encuesta de Inserción de Investigadores con Postgrado. Centro de Estudios ANIP (2018). http://www.anip.cl/
} informe-insercion-2018

1) Facultad de Economía y Negocios, Universidad Alberto Hurtado.

*Autor de correspondencia: ljimenez@uahurtado.cl 


\section{Presentación a la edición especial}

La presente edición de JOTMI es el resultado de un llamado a publicar realizado por Cristian Geldes, Christian Felzensztein y Alejandro Flores a finales del 2018. Ellos sostienen que la mayoría de los estudios en innovación están centrados en economías desarrolladas y advierten, además, que la literatura especializada de la región es relativamente escasa (Ketelhöhn y Ogliastri, 2013; Olavarrieta y Villena, 2014). También afirman que los factores que determinan la innovación en las empresas y sectores industriales de la región obedecen a dinámicas propias y no siempre son comparables a los países desarrollados (Brenes et al, 2016; Geldes et al, 2017a; Heredia et al, 2018 a).

En este sentido, la presente edición confirma en términos generales lo planteado por Geldes y col (2018). Se observa un profundo interés por el desarrollo de conocimiento específico orientado a Latinoamérica, con nuevos enfoques y nuevas realidades.

A continuación, se presentan 12 artículos que fueron seleccionados a partir de 29 trabajos provenientes de Argentina, Brasil, Chile, Colombia, Ecuador y Uruguay. Su orden y agrupación responde a tres dimensiones:

- Primero, una mirada desde la empresa, en donde se abordan las capacidades ambidiestras de las mismas, el rol de los sindicatos en la innovación empresarial, la relación de la cooperación interorganizacional en la innovación, y como la innovación se relaciona con el desempeño exportador.

- La segunda dimensión, incluye una perspectiva sectorial de la innovación, abordando temas como la gestión de portafolios innovadores en la academia, los servicios intensivos en conocimiento, los conocimientos base para la innovación en los sistemas de información y tecnológicos, y el efecto de las innovaciones de marketing en el sector de manufacturas.

- Por último, en la tercera dimensión se desarrolla un enfoque más sistémico con temas como los ecosistemas de emprendimiento, el efecto de las fallas de mercado en la inversión en innovación y los subsidios a la innovación.

Estamos muy contentos y satisfechos por la gran convocatoria que tuvimos con este número especial. Estamos seguros de estos artículos recibirán muchas citas y serán un valioso insumo para el diseño de políticas públicas en la región.
En nombre de la Facultad de Economía y Negocios de la Universidad Alberto Hurtado, queremos agradecer a todos los editores, revisores y especialmente a sus autores. Gracias por elegir a JOTMI como un medio para difundir sus investigaciones.

Santiago de Chile, diciembre, 2019.

\section{Referencias}

Brenes, E. R., Camacho, A. R., Ciravegna, L., \& Pichardo, C. A. (2016). Strategy and innovation in emerging economies after the end of the commodity boom - Insights from Latin America. Journal of Business Research, 69(10), 4363-4367. doi: 10.1016/j.jbusres.2016.03.059

Geldes, C., Felzensztein, C., \& Palacios-Fenech, J. (2017). Technological and non-technological innovations, performance and propensity to innovate across industries: The case of an emerging economy. Industrial Marketing Management, 61, 55-66. doi: 10.1016/j.indmarman.2016.10.010

Gonzalez, H., \& Jiménez, A. (2014). Inserción Laboral de Nuevos Investigadores con Grado de Doctor en Chile. Journal of Technology Management \& Innovation, 9(4), 132-148. doi: https://doi. org/10.4067/S0718-27242014000400010

Heitor, M., Horta, H., Castañón, R., Sbragia, R., \& Jiménez, A. (2014). Can Latin America Move Forward after a Lost Decade in Technical Change? ...Looking at Opportunities for Knowledge-based Change in Times of Increasing Uncertainty. Journal of Technology Management \& Innovation, 9(4), 1-19. doi: https://doi.org/10.4067/S071827242014000400001

Heredia Pérez, J. A., Geldes, C., Kunc, M. H., \& Flores, A. (2019). New approach to the innovation process in emerging economies: The manufacturing sector case in Chile and Peru. Technovation, 79, 35-55. doi: 10.1016/j.technovation.2018.02.012

Ketelhöhn, N., \& Ogliastri, E. (2013). Introduction: innovation in Latin America. Academia Revista Latinoamericana de Administración, 26(1), 12-32. doi:10.1108/arla-05-2013-0037

Olavarrieta, S., \& Villena, M. G. (2014). Innovation and business research in Latin America: An overview. Journal of Business Research, 67(4), 489-497. doi:10.1016/j.jbusres.2013.11.005

Pino, C., Felzensztein, C., Zwerg-Villegas, A. M., \& Arias-Bolzmann, L. (2016). Non-technological innovations: Market performance of exporting firms in South America. Journal of Business Research, 69(10), 4385-4393. doi:10.1016/j.jbusres.2016.03.061 
